\title{
A NOTE ON EIGENVALUES OF HECKE OPERATORS ON SIEGEL MODULAR FORMS OF DEGREE TWO
}

\author{
WINFRIED KOHNEN
}

(Communicated by William Adams)

\begin{abstract}
Let $F$ be a cuspidal Hecke eigenform of even weight $k$ on $\operatorname{Sp}_{4}(\mathbb{Z})$ with associated eigenvalues $\lambda_{m} \quad(m \in \mathbb{N})$. Under the assumption that its first Fourier-Jacobi coefficient does not vanish it is proved that the abscissa of convergence of the Dirichlet series $\sum_{m \geq 1}\left|\lambda_{m}\right| m^{-s}$ is less than or equal to $k$.
\end{abstract}

\section{INTRODUCTION}

Let $F$ be a cuspidal Hecke eigenform of weight $k \in 2 \mathbb{N}$ on $\operatorname{Sp}_{4}(\mathbb{Z})$ with associated eigenvalues $\lambda_{m} \quad(m \in \mathbb{N})$ and assume that its first Fourier-Jacobi coefficient does not vanish. Then we shall show ( $\$ 2)$ that the abscissa of convergence $\sigma_{0}$ of the Dirichlet series $\sum_{m \geq 1}\left|\lambda_{m}\right| m^{-s}$ is less than or equal to $k$. In an equivalent form, this means that $\sum_{1 \leq m \leq N}\left|\lambda_{m}\right|=O_{\varepsilon}\left(N^{k+\varepsilon}\right)$ for every $\varepsilon>0$. Note that the (straightforward) estimate $\lambda_{m}=O\left(m^{k}\right)$ only implies $\sigma_{0} \leq k+1$, while (for $F$ not in the Maass space) the Ramanujan-Petersson conjecture would predict $\sigma_{0} \leq k-\frac{1}{2}$.

Our result follows almost immediately from properties of Fourier-Jacobi coefficients of Siegel modular forms proved in [8]; however, it does not seem to have been noticed before.

\section{Estimates FOR EIGENVALUES}

For $k$ even denote by $S_{k}\left(\Gamma_{2}\right)$ the space of Siegel cusp forms of weight $k$ on $\Gamma_{2}:=\mathrm{Sp}_{4}(\mathbb{Z})$ and write $T_{k}(m)(m \in \mathbb{N})$ for the $m$ th Hecke operator on $S_{k}\left(\Gamma_{2}\right)$. By definition,

$$
T_{k}(m) F=\left.m^{2 k-3} \sum_{M \in \Gamma_{2} \backslash O_{2}(m)} F\right|_{k} M \quad\left(F \in S_{k}\left(\Gamma_{2}\right)\right),
$$

where $M$ runs through a set of representatives for the action of $\Gamma_{2}$ by leftmultiplication on the set $\mathrm{O}_{2}(\mathrm{~m})$ of integral $(4,4)$-matrices that are symplectic similitudes with scale $m$ and as usual we have put

$$
\left(\left.F\right|_{k} M\right)(Z)=\operatorname{det}(C Z+D)^{-k} F\left((A Z+B)(C Z+D)^{-1}\right)
$$

Received by the editors May 20, 1990 and, in revised form, August 5, 1990.

1980 Mathematics Subject Classification (1985 Revision). Primary 11F46. 
$\left(M=\left(\begin{array}{ll}A & B \\ C & D\end{array}\right), Z \in \mathfrak{H}_{2}=\right.$ Siegel upper half-space of degree 2).

Let $F \in S_{k}\left(\Gamma_{2}\right)$ be a nonzero eigenfunction of all Hecke operators, and denote by $\lambda_{m}$ the eigenvalue of $F$ with respect to $T_{k}(m)$. We let $\sigma_{0}$ be the abscissa of convergence of the Dirichlet series $\sum_{m \geq 1}\left|\lambda_{m}\right| m^{-s}$. Note that by classical results on Dirichlet series, one has $\sigma_{0}=\inf \left\{\alpha \in \mathbb{R}\left|\sum_{1 \leq m \leq N}\right| \lambda_{m} \mid=\right.$ $\left.O\left(N^{\alpha}\right)\right\}$ (supposing that $\sum_{m>1}\left|\lambda_{m}\right|$ diverges).

Counting the number of left cosets in $O_{2}(m)$ (cf. $[1,4,9]$ ) one easily obtains the estimate $\lambda_{m}=O\left(m^{k}\right)$ and hence $\sigma_{0} \leq k+1$.

This can be improved to

$$
\lambda_{m}=O_{\varepsilon}\left(m^{k-1 / 2+\varepsilon}\right) \quad(\varepsilon>0)
$$

(hence $\sigma_{0} \leq k+\frac{1}{2}$ ) if one combines on the one-hand side Kitaoka's estimate

$$
a(T)=O_{\varepsilon}\left((\operatorname{det} T)^{k / 2-1 / 4+\varepsilon}\right) \quad(\varepsilon>0),
$$

where $a(T)$ is the $T$ th Fourier coefficient of $F$ and $T$ is any positive definite half-integral $(2,2)$-matrix (cf. [6]), and on the other hand Andrianov's results relating the spinor zeta function $Z_{F}(s)$ of $F$ to partial zeta functions of the form $\sum_{m \geq 1} a(m T) m^{-s}$ (cf. [1]); recall that if one puts $Z_{F, p}(X):=$ $1-\lambda_{p} X+\left(\lambda_{p}^{2}-\lambda_{p^{2}}-p^{2 k-4}\right) X^{2}-\lambda_{p} p^{2 k-3} X^{3}+p^{4 k-6} X^{4} \quad(p$ a prime), then $Z_{F}(s)=\prod_{p} Z_{F, p}\left(p^{-s}\right)^{-1}=\zeta(2 s-2 k+4)^{-1} \sum_{m \geq 1} \lambda_{m} m^{-s}$ [1]. Also by [1], $\tilde{Z}_{F}(s)=(2 \pi)^{-2 s} \Gamma(s) \Gamma(s-k+2) Z_{F}(s)$ has a meromorphic continuation to $\mathbb{C}$ and is invariant under $s \mapsto 2 k-2-s$.

If $F$ is in the Maass subspace $S_{k}\left(\Gamma_{2}\right)^{*} \subset S_{k}\left(\Gamma_{2}\right)$, then $Z_{F}(s)=\zeta(s-k+1)$ $\times \zeta(s-k+2) L_{f}(s)$ where $f$ is an elliptic cusp form of weight $2 k-2$ [2]. From this one can easily conclude that $\lambda_{m}>0$, and therefore $\sigma_{0}=k$ for such $F$. Note that by $[3,10]$ one has $F \in S_{k}\left(\Gamma_{2}\right)^{*}$ iff $\widetilde{Z}_{F}(s)$ has poles.

On the other hand, for $F$ in the orthogonal complement of the Maass space, one expects that the generalized Ramanujan-Petersson conjecture holds which predicts that the roots of the polynomial $Z_{F, p}(X)$ are of absolute value $p^{-k+3 / 2}$ for all $p$.

The Ramanujan-Petersson conjecture would imply the estimate

$$
\lambda_{m}=O_{\varepsilon}\left(m^{k-3 / 2+\varepsilon}\right) \quad(\varepsilon>0)
$$

and hence $\sigma_{0} \leq k-\frac{1}{2}$, but at least at present a proof of it seems to be out of range.

\section{STATEMENT OF RESUlt AND PROOF}

Recall that the function $F$ has a Fourier-Jacobi expansion of the form

$$
F(Z)=\sum_{m \geq 1} \varphi_{m}(\tau, z) e^{2 \pi i m \tau^{\prime}} \quad\left(Z=\left(\begin{array}{cc}
\tau & z \\
z & \tau^{\prime}
\end{array}\right)\right),
$$

where the $\varphi_{m}$ 's are Jacobi cusp forms of weight $k$ and index $m$ [2]. We shall 
prove:

Theorem. Let $F \in S_{k}\left(\Gamma_{2}\right)$ be a nonzero Hecke eigenform with eigenvalues $\lambda_{m}$ w.r.t. $T_{k}(m)$ and suppose that its first Fourier-Jacobi coefficient $\varphi_{1}$ does not vanish. Denote by $\sigma_{0}$ the abscissa of convergence of the Dirichlet series $\sum_{m \geq 1}\left|\lambda_{m}\right| m^{-s}$. Then $\sigma_{0} \leq k$.

Remark. The condition " $\varphi_{1} \neq 0$ " holds for (at least) all nonzero Hecke eigenforms of weight $k$ with $k$ in the range $10 \leq k \leq 32$ (cf. [12]). Being optimistic, one could hope that it is always satisfied (cf. [11]).

Proof of Theorem. The assertion is a more or less immediate consequence of the results proved in [8]. Let $J_{k, m}^{\text {cusp }}$ be the space of Jacobi cusp forms of weight $k$ and index $m$ and write $\langle\rangle=,\langle,\rangle_{m, k}$ for the usual Petersson scalar product on $J_{k, m}^{\text {cusp }}$ [2]. Let $G$ be any function in the Maass space $S_{k}\left(\Gamma_{2}\right)^{*}$ and write $\psi_{m}$ for its $m$ th Fourier-Jacobi coefficient. Then according to $[5,8]$ we have

$$
\left\langle\varphi_{m}, \psi_{m}\right\rangle=\left\langle\varphi_{1}, \psi_{1}\right\rangle \lambda_{m} .
$$

Since $\varphi_{1} \neq 0$ by assumption and the map $G \mapsto \psi_{1}$ is an isomorphism of $S_{k}\left(\Gamma_{2}\right)^{*}$ onto $J_{k, 1}^{\text {cusp }}$ [2] we can find $G \in S_{k}\left(\Gamma_{2}\right)^{*}$ with $\left\langle\varphi_{1}, \psi_{1}\right\rangle \neq 0$, and with this choice of $G$ we can write

$$
\lambda_{m}=\left\langle\varphi_{m}, \psi_{m}\right\rangle /\left\langle\varphi_{1}, \psi_{1}\right\rangle .
$$

By the Cauchy-Schwarz inequality we have

$$
\left|\left\langle\varphi_{m}, \psi_{m}\right\rangle\right| \leq\left\|\varphi_{m}\right\| \cdot\left\|\psi_{m}\right\| \leq \frac{1}{2}\left(\left\|\varphi_{m}\right\|^{2}+\left\|\psi_{m}\right\|^{2}\right) .
$$

On the other hand, as proved in [8], the Dirichlet series $\sum_{m \geq 1}\left\|\varphi_{m}\right\|^{2} m^{-s}$ (resp. $\quad \sum_{m \geq 1}\left\|\psi_{m}\right\|^{2} m^{-s}$ )-originally only defined in the half-plane $\operatorname{Re}(s)>$ $k+1$-have meromorphic continuations to $\operatorname{Re}(s) \geq k$ with simple poles at $s=k$ as its only singularities. Therefore, by a theorem of Landau the abscissa of convergence of these Dirichlet series is equal to $k$. Hence we deduce $\sigma_{0} \leq k$.

Remarks. (i) An interesting question seems to be whether the estimate $\left\|\varphi_{m}\right\|^{2}=$ $O_{\varepsilon}\left(m^{k-1+\varepsilon}\right)$ would hold for any Hecke eigenform $F$ in $S_{k}\left(\Gamma_{2}\right)$ (from the above discussion one sees that it is certainly satisfied for $\left.F \in S_{k}\left(\Gamma_{2}\right)^{*}\right)$. Note that under the condition " $\varphi_{1} \neq 0$ ", this would imply that $\lambda_{m}=O_{\varepsilon}\left(m^{k-1+\varepsilon}\right)$. To answer the above question one naturally seems to be led to a closer study of the Fourier-Jacobi coefficients of the Poincaré-type series introduced by Klingen in [7].

(ii) S. Böcherer informs the author that very recently J. S. Li (using results of $\mathrm{R}$. Howe) proved the estimate $\left|\lambda_{p}\right| \leq 4 p^{k-1}$ ( $p$ a prime).

\section{REFERENCES}

1. A. N. Andrianov, Euler products corresponding to Siegel modular forms of genus 2 , Russian Math. Surveys 29 (1974), 45-116.

2. M. Eichler and D. Zagier, The theory of Jacobi forms, Progr. Math., vol. 55, Birkhäuser, Boston, 1985. 
3. S. A. Evdokimov, A characterization of the Maass space of Siegel cusp forms of degree 2, Math. USSR-Sb. 112 (1980), 133-142. (Russian)

4. E. Freitag, Siegelsche Modulfunktionen, Grundlehren Math. Wiss., vol. 254, Springer, New York, 1983.

5. V. A. Gritsenko, The action of modular operators on the Fourier-Jacobi coefficients of modular forms, Math. USSR-Sb. 47 (1984), 237-267.

6. Y. Kitaoka, Fourier coefficients of Siegel cusp forms of degree two, Nagoya Math. J. 93 (1984), 149-171.

7. H. Klingen, Über Kernfunktionen für Jacobiformen und Siegelsche Modulformen, Math. Ann. 285 (1989), 405-416.

8. W. Kohnen and N.-P. Skoruppa, A certain Dirichlet series attached to Siegel modular forms of degree two, Invent. Math. 95 (1989), 541-558.

9. A. Krieg, Das Vertauschungsgesetz zwischen Hecke-Operatoren und dem Siegelschen $\Phi$ Operator, Arch. Math. 46 (1986), 323-329.

10. T. Oda, On the poles of Andrianov L-functions, Ann. of Math. (2) 256 (1981), 323-340.

11. N.-P. Skoruppa, Developments in the theory of Jacobi forms, Automorphic Functions and Their Applications (N. Kuznetsov and V. Bykovsky, eds.), The USSR Academy of Sciences, Khabavovsk, 1990, pp. 167-185.

12. __ Computations of Siegel modular forms of genus two, preprint, MPI Bonn, 1990.

Max-Planck-Institut für Mathematik, Gottfried-Claren-Str. 26, 5300 Bonn 3, FedERAL REPUBLIC OF GERMANY 